\title{
快速溶剂交换法制备疏水性二氧化硅气凝胶及其 负载有机苂光探针的应用研究
}

\author{
王亚飞 $\dagger$ 张 涛 \\ 王双青*,a 杨国强*,a \\ ( ${ }^{a}$ 中国科学院化学研究所光化学重点实验室 中国科学院大学 北京 100190) \\ ( ${ }^{b}$ 中国科学院深圳先进技术研究院先进材料中心 深圳 518055)
}

\begin{abstract}
摘要 气凝胶是流动相为气体的凝胶材料, 具有低密度、高孔隙率的特性, 有着广泛的应用前景. 本工作开发了一种快 速溶剂交换法，利用原位二级萃取的原理，通过乙醇-二氯甲烷一水三种溶剂间互溶-不互溶的特性实现了气凝胶制备 中的快速溶剂交换，结合疏水性处理和减压干燥得到了疏水性二氧化硅气凝胶. 所得到的气凝胶密度低，与水的接触 角达到 $155.8^{\circ}$. 该气凝胶可以负载多种有机苂光探针染料, 可以有效避免由于探针分子聚集而引起的苂光猝灭, 将有 可能使有机苂光探针在更广泛的领域得到实际应用.
\end{abstract}

关键词 气凝胶; 有机苂光探针; 制备; 快速溶剂交换法; 应用

\section{Preparation of Hydrophobic $\mathrm{SiO}_{2}$ Aerogel by Rapid Solvents Exchange Method and Its Application Loaded with Organic Fluorescence Probe}

\author{
Wang, Yafei ${ }^{\dagger, a} \quad$ Zhang, Tao ${ }^{\dagger, a, b} \quad$ Guo, Xudong ${ }^{a} \quad$ Hu, Rui ${ }^{a}$ \\ Wang, Shuangqing ${ }^{*, a} \quad$ Yang, Guoqiang ${ }^{*, a}$ \\ ( ${ }^{a}$ Key Laboratory of Photochemistry, Institute of Chemistry, University of Chinese Academy of Sciences, \\ Chinese Academy of Sciences, Beijing 100190) \\ ( ${ }^{b}$ Center of Advanced Material Research, Shenzhen Institutes of Advanced Technology, Chinese Academy of Sciences, \\ Shenzhen 518055)
}

\begin{abstract}
Aerogel is a kind of gel materials, of which the fluid phase is gas. Aerogel has the characteristics of low density and high porosity, and has a wide application prospects. In this work, a rapid solvents exchange method was developed, and using in-situ secondary extraction, rapid solvents exchange in aerogel preparation was achieved through the miscibility and immiscibility of ethanol-dichloromethane-water. Combined with hydrophobic treatment and vacuum drying, hydrophobic silica aerogel was obtained. The aerogel had a low density and a contact angle with water of $155.8^{\circ}$. The aerogel can load various organic fluorescent probe dyes, which can effectively avoid the fluorescence quenching caused by the aggregation of the probe molecules, which it will expand the practical application range of the organic fluorescent probes in wider fields.

Keywords aerogel, organic fluorescence probe, preparation, rapid solvents exchange method, application
\end{abstract}

气凝胶是一种固体物质形态, 是流动相为气体的凝 胶材料. 它一般是在不改变凝胶的微观结构的情况下去 除凝胶中的溶剂得到的. 目前研究得比较广泛的气凝胶 体系是二氧化硅气凝胶、有机气凝胶、碳气凝胶等. 在
气凝胶的制备过程中较好地保留了凝胶的微孔结构, 所 以气凝胶一般具有空隙率高、比表面积大、密度低的特 点. 独特的微观结构赋予了气凝胶一系列特异的性质， 如低折射率、低热导率、低声阻抗以及良好的比强度等.

\footnotetext{
* Corresponding authors. E-mail: g1704@iccas.ac.cn; gqyang@iccas.ac.cn

Received August 5, 2018; revised September 6, 2018; published online September 26, 2018.

Project supported by the National Natural Science Foundation of China (Nos. 21873106, 21627802)

国家自然科学基金(Nos. 21873106, 21627802)资助项目

†共同第一作者(These authors contributed equally to this work).
} 
气凝胶的这些理化特性, 使得它在催化剂及催化剂载 体、绝热材料、声阻抗耦合材料、超级电容器等领域都 有很大的实用价值或应用潜力 ${ }^{[1-3]}$.

气凝胶的优良性能或可以在某些领域, 例如作为绝 热材料, 对传统的材料形成革命性的冲击或者替代 ${ }^{[4,5]}$. 气凝胶复杂的制备工艺和高昂的成本大大限制了气凝 胶的进一步推广和应用 ${ }^{[6]}$. 气凝胶的制备方法主要包括 超临界干燥法、蒸发(包括常压和减压)干燥法和冷冻干 燥法, 而目前的制备工艺各自都存在一定的局限性, 难 以实现气凝胶的大规模生产, 有必要对气凝胶的制备方 法和工艺进行深入的研究, 以克服传统制备方法的不 足 $^{[7,8]}$.

有机荧光探针是一类受环境(温度、极性、 $\mathrm{pH}$ 值、 粘度等)变化诱发的有机分子发光光谱发生显著改变的 有机化合物, 通过发光光谱的改变可以定性或定量检测 环境因素的改变量或变化量. 相对于传统的检测方法, 有机苂光探针具有尺度小的特点, 能达到较高的空间分 辨率, 同时具有高的灵敏性和选择性. 部分有机分子具 有好的生物兼容性, 有机苂光分子被广泛应用于细胞和 生物成像, 开启了细胞生物学的新纪元. 这些有机荧光 探针已经在温度检测 ${ }^{[9,10]} 、 \mathrm{pH}$ 值检测 ${ }^{[11]}$ 、生物医学检 测 ${ }^{[12 ~ 19]}$ 等多个领域取得较大的突破, 部分苂光探针已 经开始实际应用.

有机苂光探针的使用需要在一定的环境中, 大多是 在有机溶剂体系、水体系中, 分散在固体薄膜或凝胶中. 在固体状态时, 有机荧光探针所使用的有机化合物分子 的发光性质和化合物本身的聚集状态有很大关系, 大部 分探针分子在聚集状态的发光会被自身猝灭. 考虑到气 凝胶的多孔性能, 将有机苂光探针分散在多孔性的气凝 胶中, 可以有效避免由于探针分子的聚集而引起的荧光 猝灭.

本工作使用一种快速溶剂交换法, 结合疏水处理及 减压干燥方法, 显著减少了常压干燥制备 $\mathrm{SiO}_{2}$ 气凝胶 的时间与溶剂使用量. 所得气凝胶具有疏水性能良好, 且对多种苂光物质良好的负载能力 ${ }^{[20,21]}$. 将气凝胶作为 有机苂光探针的载体, 可以拓展有机苂光探针的应用领 域, 有望应用于微流体示踪、温度指示及痕量物质检测 等领域，也拓展了气凝胶的实际应用.

\section{1 结果与讨论}

\section{1 二氧化硅凝胶的制备}

以正硅酸乙酯(TEOS) 作为硅源, 直接在醇溶液中 经两步催化溶胶一凝胶法制备二氧化硅凝胶. 将一定量 的 TEOS、乙醇、去离子水、氢氟酸(HF)水溶液加热回 流搅拌均匀, 得到酸性澄清液 A. 另配备一定量含有乙
醇、去离子水、氨水 $\left(\mathrm{NH}_{3} \cdot \mathrm{H}_{2} \mathrm{O}\right)$ 的碱性澄清液 B. 室温剧 烈搅拌下将溶液 $\mathrm{B}$ 加入到溶液 $\mathrm{A}$, 得到透明的二氧化硅 凝胶.

在酸性条件下, 体系中主要发生的是水解反应, 加 入的水量低于完全反应时的化学计量比, 溶液 $\mathrm{A}$ 保持澄 清的液体状态; 而碱性条件有利于缩聚的发生，并且补 加了足量的水后, 有利于反应的正向进行. 酸催化水解 与碱催化聚合这两部分反应并不是绝然分开的, 前驱体 TEOS 中的四个官能团并不会完全水解后才开始发生缩 聚反应, A 液中发生水解的同时也会有缩聚的发生, 加 入 $\mathrm{B}$ 液后未水解的烷氧基也可以继续水解. 图 1 给出了 TEOS 形成二氧化硅凝胶的过程, 包括硅酸酯的水解以 及硅醇与硅醇、硅醇与硅脂的缩聚. 其中缩聚反应形成 的 $\mathrm{Si}-\mathrm{O}-\mathrm{Si}$ 键是凝胶网络形成的基础, 凝胶网络生长 到占据整个液体空间时, 就形成二氧化硅凝胶.

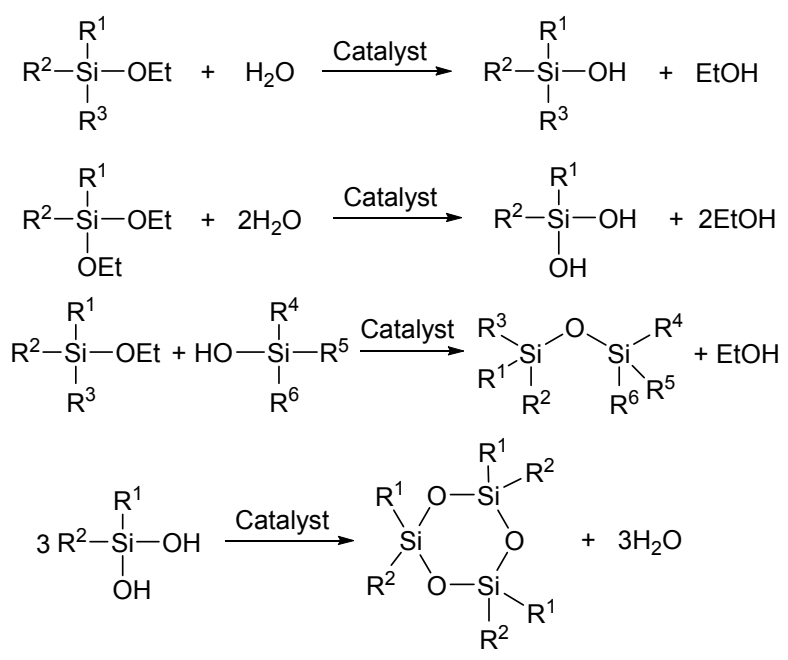

图 1 由正硅酸乙酯为硅源制备二氧化硅凝胶

Figure 1 Preparation of silica gel from tetraethoxysilane as silicon source

\section{2 快速溶剂交换}

溶剂交换是指采用表面张力较小的溶剂替换凝胶 中的溶剂(水、乙醇), 以便在后续干燥过程中得到具有 良好多孔结构的气凝胶. 一般的溶剂交换过程就是采用 大量的溶剂多次浸泡凝胶, 从而将凝胶中原有的溶剂逐 渐替换掉. 溶剂置换后, 还有利于保持气凝胶良好的物 理形状, 减少气凝胶的龟裂或变形. 为保证溶剂交换效 果, 长时间、多次数、大体积地使用新鲜的溶剂是必不 可少的，进而增加了制备气凝胶的时间及材料的成本. 多级萃取及反萃取技术可以高效地将目标物质富集、分 离 ${ }^{[22,23]}$. 凝胶中溶剂的交换实际上也是一个将母液从凝 胶中分离出来的过程, 结合多级萃取与反萃取技术，设 计了原位二级溶剂萃取工艺, 实现了溶剂的快速置换. 图 2 给出了二氯甲烷-水体系的原位二级萃取系统原理. 


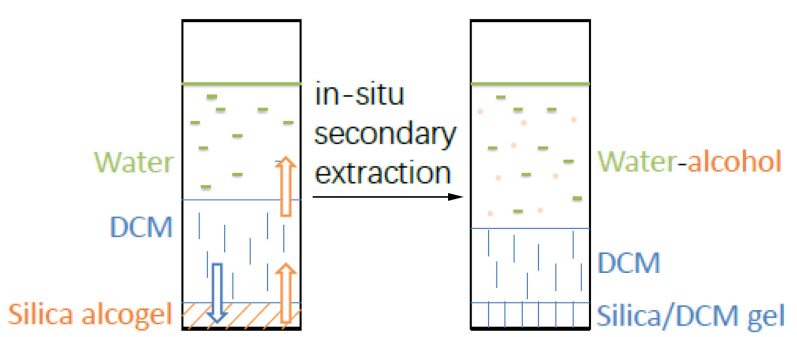

图 2 二氯甲烷一水体系的原位二级萃取原理图

Figure 2 In situ secondary extraction from dichloromethanewater system

如图 2 所示, 二氯甲烷(DCM)与乙醇互溶而与水的 相互溶解能力很小, 且溶解于二氯甲烷的乙醇可被水反 萃取进入水相. 二氯甲烷的密度大于水, 这使得二氯甲 烷可以处于下层, 从而隔绝了水相与凝胶相的直接接 触, 这一二级萃取操作可以直接在制备凝胶的模具中进 行. 在二级萃取实验中, 完成老化的凝胶放置在模具底 层, 依次向模具中加入二氯甲烷、去离子水. 乙醇迅速 上浮进入二氯甲烷相, 然后转移入水相中, 同时二氯甲 烷进入凝胶相中. 对于厚度为 $5 \mathrm{~mm}$ 左右的样品, 经过 2 4 h 即可基本完成二级萃取. 该方法利用与乙醇有较 好互溶度但彼此不相溶的两种溶剂, 其中一种溶剂与二 氧化硅醇凝胶直接接触, 将凝胶中的乙醇置换出来的同 时自己进入凝胶内部; 另一种溶剂则将乙醇从前一种溶 剂中不断反萃取出来. 通过这个方法可以方便、快捷、 低成本地实现凝胶体系中溶剂的交换.

\section{3 疏水性处理与干燥}

向完成二级萃取溶剂交换的二氧化硅凝胶中加入 足量的疏水处理剂三甲基氯硅烷(TMCS)、六甲基二硅 氮烷(HMDS)、六甲基二硅氧烷(HMDSO)进行疏水化处 理, 得到的气凝胶分别标记为 A-TMCS、A-HMDS 和 A-HMDSO, 未经疏水处理直接干燥的样品记为 A-O. 室温静置 $8 \mathrm{~h}$ 左右, 让疏水处理剂进入凝胶内部与凝胶 网络表面的硅羟基完成反应. 三种疏水处理剂每个硅原 子直接与三个甲基相连, 具有一个可与硅羟基反应的位 点. 凝胶中大部分的硅羟基被疏水处理剂封端后, 可防 止干燥过程中硅羟基彼此之间脱水缩合使凝胶发生不 可逆的收缩.

干燥是将凝胶中的溶剂去除得到固体的气凝胶, 通 常使用较多的有常压干燥法和减压干燥法两种方 式 ${ }^{[24,25]}$, 所得到的气凝胶的密度基本相同, 但减压干燥 有利于加速干燥过程. 为了缩短气凝胶的制备时间, 同 时, 考虑到二氯甲烷溶剂的回收, 本工作采用减压干燥 的方法得到了性能优良的疏水性二氧化硅气凝胶.

\section{4 二氧化硅气凝胶的表征}

密度是衡量气凝胶的重要参数, 与密度相关的是孔 隙率, 这通常与气凝胶的制备和后处理过程相关. 表 1
给出了不同疏水处理剂制备的主要性质参数. 气凝胶的 块体密度是气凝胶的最根本数据, 由气凝胶的质量及表 观体积计算得到. 数据表明, 三甲基氯硅烷对凝胶表面 羟基的封端效果较好, 气凝胶的块体密度可以低到

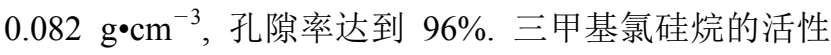
最强, 极易与硅羟基反应, 而六甲基二硅氮烷及六甲基 二硅氧烷与硅羟基的反应对溶剂、反应温度、催化剂等 条件比较敏感，在室温及二氯甲烷溶液中后两种疏水处 理剂与硅羟基的反应效率并不高. 通过氮气吸附-脱附 测试测定了三甲基氯硅烷处理和未处理气凝胶的比表 面积，处理后的比表面积明显提高，其原因是三甲基氯 硅烷处理后, 凝胶网络表面羟基上的活泼氢被三甲基硅 基取代, 可以有效避免在干燥过程中因硅羟基相互接触 而进一步缩合，从而保留了更加丰富的孔结构.

表 1 不同疏水处理制备的气凝胶的主要性质 Table 1 Aerogel's properties prepared from different hydrophobic treatment

\begin{tabular}{lccc}
\hline 处理方式 & $\rho_{\mathrm{b}} /\left(\mathrm{g} \cdot \mathrm{cm}^{-3}\right)$ & $\varepsilon^{a} / \%{ }^{[26]}$ & $S /\left(\mathrm{m}^{2} \cdot \mathrm{g}^{-1}\right)$ \\
\hline A-TMCS & 0.082 & 96.0 & $8.91 \times 10^{2}$ \\
A-HMDS & 0.135 & 93.4 & - \\
A-HMDSO & 0.171 & 91.7 & - \\
A-O & 0.343 & 83.3 & $8.51 \times 10^{2}$ \\
\hline${ }^{a} \varepsilon=\left(1-\rho_{\mathrm{b}} / \rho_{\mathrm{s}}\right) \times 100 \%$, 该类气凝胶的骨架密度 $\rho_{\mathrm{s}}$ 以 $2.06 \mathrm{~g} \cdot \mathrm{cm}^{-3}$ 计算. \\
其中, $\rho_{\mathrm{b}}$ 为块体密度, $\varepsilon$ 为孔隙率, $\rho_{\mathrm{s}}$ 为骨架密度, $S$ 为比表面积.
\end{tabular}

采用快速溶剂交换法制得的气凝胶是一种白色半 透明块体, 扫描电镜显示(图 3)所得的 A-TMCS 气凝胶 由纳米级的球状聚集体构成, 球状聚集体的粒径在 5 $20 \mathrm{~nm}$ 之间. 其特征尺寸远小于可见光波长, 整体结构 比较均一, 这也是它具有一定透明度的主要原因.

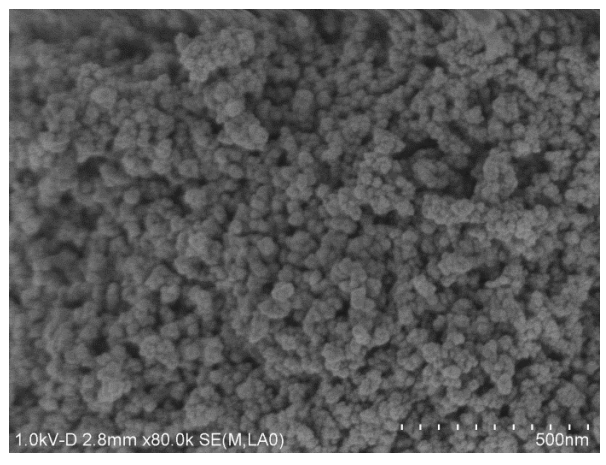

图 3 气凝胶 A-TMCS 的扫描电子显微结构图

Figure 3 SEM image of aerogel A-TMCS

如图 4 所示, 经测定, 制备的 A-TMCS 气凝胶接触 角达到了 $155.8^{\circ}$, 是一种超疏水的固体材料. 经过快速 溶剂萃取得到的湿凝胶经过量 TMCS 处理后, 其网络表 面极性硅羟基被具有良好疏水性的三甲基硅基取代，使 气凝胶的表面能显著降低, 与水分子的作用力大大减 弱, 进而有可能得到较大的接触角. 


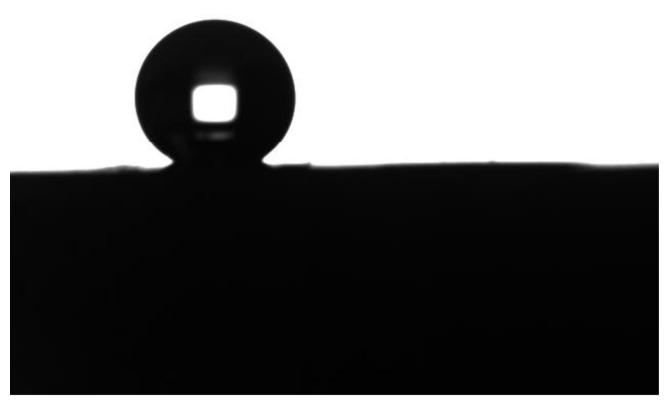

图 4 气凝胶 A-TMCS 的接触角

Figure 4 Contact angle of aerogel A-TMCS

\section{5 二氧化硅气凝胶负载有机苂光探针}

二氧化硅气凝胶具有高的比表面积与大的孔隙度, 经过疏水剂处理后具有一定的亲油性, 有利于各种常用 溶剂及有机功能材料 (如有机苂光探针染料) 的吸 附 ${ }^{[20,21]}$. 有机苂光探针已经在多个领域得到应用, 在某 些情况下, 需要使用固体状态的探针染料. 但是在固体 状态时, 除了部分特殊分子结构具有聚集诱导发光特性

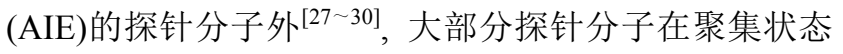
的发光会被自身猝灭. 考虑到气凝胶的多孔性能, 将有 机苂光探针分散在多孔性的气凝胶中, 将可以有效避免 由于探针分子的聚集而引起的荧光猝灭, 进而拓展有机 荧光探针的应用范围 ${ }^{[31]}$.

将制备的疏水性二氧化硅气凝胶经过高速粉碎机 粉碎, 得到微米至亚微米的二氧化硅气凝胶粉末. 将气 凝胶粉末加入到有机苂光探针荧光素的乙醇溶液, 充分 吸附后去除乙醇溶剂, 得到负载有有机荧光探针的二氧 化硅气凝胶. 负载有机荧光染料的疏水二氧化硅气凝胶 可用于物品上遗留指纹的收集, 将有可能在刑事侦查领 域得到应用. 与手指接触后, 人体分泌的油性物质会转 移到物体上, 这些油性物质对亲油的二氧化硅气凝胶具 有很好的亲和力, 与之接触时能很好地粘附二氧化硅气 凝胶粉末. 将负载有荧光素的二氧化硅气凝胶粉末吹扫 于手指接触处, 在紫外灯照射下, 气凝胶中吸附的有机 荧光染料会发出特定波长的光, 可以得到明显的指纹图 案(图 5). 图 5a 中的指纹是手指直接与载玻片接触留下 的指纹, 图 $5 b$ 中是手指触摸过前额后留下的指纹, 与前 额接触后会残留更多的油脂, 因此相同处理条件下会粘 附更多的气凝胶粉末.

\section{2 结论}

利用原位二级萃取的原理, 通过乙醇-二氯甲烷-水 三种溶剂间互溶一不互溶的特性实现了气凝胶制备中的 快速溶剂交换, 结合疏水性处理和减压干燥得到了疏水 性二氧化硅气凝胶. 采用快速溶剂交换法制备二氧化硅 气凝胶可以大大节省制备时间, 减少溶剂的使用量, 可
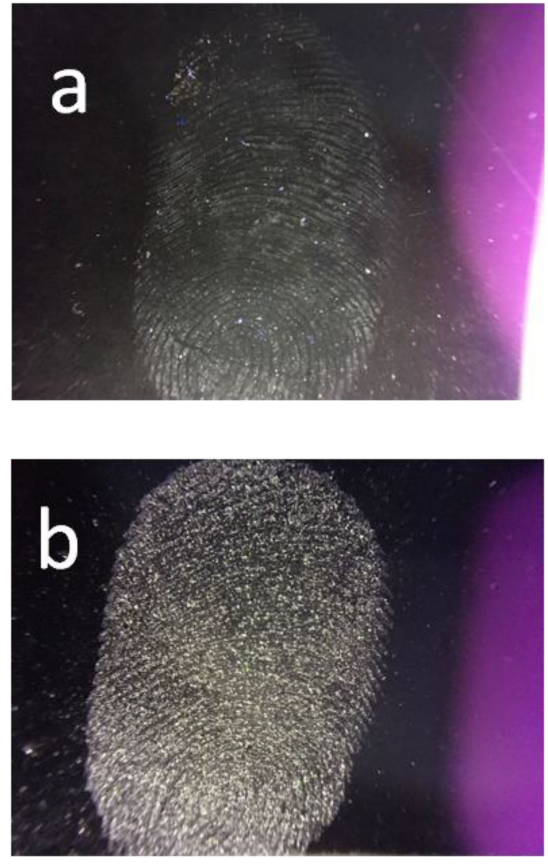

图 5 负载苂光素分子的二氧化硅气凝胶粉末在指纹收集方 面的应用

Figure 5 Application of silica aerogel loaded with fluorescein molecule in fingerprint collection

实现快速低成本地制备二氧化硅气凝胶. 所得到的疏水 性气凝胶的密度低, 与水的接触角达到 $155.8^{\circ}$, 是一种 超疏水的固体材料. 该气凝胶可以负载多种有机苂光探 针染料, 可以有效避免由于探针分子的聚集而引起的荧 光猝灭, 进而拓展有机苂光探针的应用范围, 将有可能 使有机苂光探针在更广泛的领域得到实际应用.

\section{3 实验部分}

\section{1 气凝胶制备典型步骤}

在匀速搅拌下, 依次向 $100 \mathrm{~mL}$ 烧杯中加入 $12.5 \mathrm{~mL}$ 正硅酸乙酯(TEOS)、 $25.0 \mathrm{~mL}$ 无水乙醇 $(\mathrm{EtOH}) 、 1.0 \mathrm{~mL}$ 去离子水及 $18 \mu \mathrm{L} \mathrm{HF}$ 水溶液(质量浓度 $40 \%$ ), 混合均匀 后 $\mathrm{pH}$ 值约为 3.5 , 将此混合液记为溶液 A. $60{ }^{\circ} \mathrm{C}$ 水浴条 件下, 搅拌并回流反应 $90 \mathrm{~min}$, 摚拌下冷却, 此过程中 溶液保持澄清状态. 向另一 $25 \mathrm{~mL}$ 烧瓶中加入 $15.0 \mathrm{~mL}$ 无水乙醇、 $2.5 \mathrm{~mL}$ 去离子水及 $10 \mu \mathrm{L}$ 氨水 $\left(\mathrm{NH}_{3} \cdot \mathrm{H}_{2} \mathrm{O}\right.$, 质 量浓度 $25 \%$ ), 混合均匀后备用, 记为溶液 B. 室温剧烈 搅拌下将溶液 B 加入溶液 A 中, 可形成透明的二氧化硅 凝胶. 凝胶形成后, 移入预升温至 $50{ }^{\circ} \mathrm{C}$ 的烘箱中老化 3 h. 按照图 2 所示, 向容器中加入二氯甲烷和去离子水, 经过大约 $2 \sim 4 \mathrm{~h}$ 的二级萃取操作后, 移除水相与二氯甲 烷相. 疏水处理或者不疏水处理后真空干燥, 得到厚度 约为 $5 \mathrm{~mm}$ 的不规则块状气凝胶, 再经高速粉碎机粉碎, 得到二氧化硅气凝胶粉末. 


\section{2 气凝胶吸附有机荧光探针}

向 $10 \mathrm{~mL} 1.0 \times 10^{-6} \mathrm{~mol} \cdot \mathrm{L}^{-1}$ 的苂光素乙醇溶液中加 入 $100 \mathrm{mg}$ 二氧化硅气凝胶粉末, 超声震荡后静置, 自然 挥发可得负载有有机染料分子的二氧化硅气凝胶. 苂光 素分散在气凝胶中和在溶液中的发光强度相当.

\section{3 仪器与试剂}

形貌分析测试采用日立 SU8010 扫描电子显微镜, 接触角测试采用 KRUSS DSA100 接触角测量仪, 氮气 吸附-脱附测试采用 NOVA 3200e 比表面物理化学吸附 仪进行测试.

正硅酸乙酯(国药集团化学试剂有限公司, 分析 纯)、三甲基氯硅烷(国药集团化学试剂有限公司, 分析 纯)、六甲基二硅氮烷(ACROS, 98\%)、六甲基二硅氧烷 (ACROS，98\%), 其他试剂如无水乙醇、二甲基甲酰胺 (分析纯)、二氯甲烷(分析纯)、氨水 $(25 \% \sim 28 \%) 、 H F$ 水 溶液( $40 \%)$ 等均购自北京化学试剂公司.

辅助材料(Supporting Information) 厚度约为 $5 \mathrm{~mm}$ 的 不规则块状气凝胶图片, 尼罗红固体和负载尼罗红的气 凝胶在紫外灯下的发光对比. 这些材料可以免费从本刊 网站(http://sioc-journal.cn/)上下载.

\section{References}

[1] Wang, X. C.; Li, J. Z.; Li, G. Y.; Wang, J.; Zhang, X. T.; Guo, Q. Acta Phys.-Chim. Sin. 2017, 33, 2141 (in Chinese) (王叙春, 李金泽, 李广勇, 王锦, 张学同, 郭强, 物理化学学报, 2017, 33, 2141.)

[2] Cuce, E.; Cuce, P. M.; Wood, C. J.; Riffat, S. B. Renewable Sustainable Energy Rev. 2014, 34, 273.

[3] Gurav, J. L.; Jung, I.-K.; Park, H.-H.; Kang, E. S.; Nadargi, D. Y. J. Nanomater. 2010, 409310.

[4] Aegerter, M. A.; Leventis, N.; Koebel, M. M. Aerogels Handbook, Springer, New York, 2011.

[5] Hüsing, N.; Schubert, U. Angew. Chem., Int. Ed. 1998, 37, 22.

[6] Jia, G. H; Li, Z; Liu, P; Jing, Q. S. J. Non-Cryst. Solids 2018, 482, 192.

[7] Wei, T.-Y.; Chang, T.-F.; Luw, S.-Y.; Chang, Y.-C. J. Am. Ceram. Soc. 2007, 90, 2003

[8] Chen, Q. F.; Wang, H.; Sun, L. Y. Materials 2017, 10, 435.

[9] Feng, J.; Tian, K. J.; Hu, D. H.; Wang, S. Q.; Li, S. Y.; Zeng, Y.; Li, Y.; Yang, G. Q. Angew. Chem., Int. Ed. 2011, 50, 8072.
[10] Liu, J.; Guo, X. D.; Hu, R.; Xu, J.; Wang, S. Q.; Li, S. Y.; Li, Y.; Yang, G. Q. Anal. Chem. 2015, 87, 3694.

[11] Cao, L. X.; Li, X. Y.; Wang, S. Q.; Li, S. Y.; Li, Y.; Yang, G. Q. Chem. Commun. 2014, 50, 8787.

[12] Li, S.; Hu, R.; Yang, C. L.; Zhang, X.; Zeng, Y.; Wang, S. Q.; Guo, X. D.; Li, Y.; Cai, X. P.; Li, S. R.; Han, C. W.; Yang, G. Q. Biosens. Bioelectron. 2017, 98, 325.

[13] Hu, D. H.; Zhang, T.; Li. S. Y.; Yu, T. J.; Zhang, X. H.; Hu, R.; Feng, J.; Wang, S. Q.; Liang, T. L.; Chen, J. M.; Sobenina, L. N.; Trofimov, B. A.; Li, Y.; Ma, J. S.; Yang, G. Q. Nat. Commun. 2018, 9, 362 .

[14] Kolanowski, J. L.; Liu, F.; New, E. J. Chem. Soc. Rev. 2018, 47, 195.

[15] Sinkeldam, R. W.; Greco, N. J. Tor, Y. Chem. Rev. 2010, 110, 2579.

[16] Zhao, Z. S.; Guo, X. D.; Li, S. Y.; Yang, G. Q. Acta Chim. Sinica 2016, 74, 593 (in Chinese). (赵振盛, 郭旭东, 李沙瑜, 杨国强, 化学学报, 2016, 74, 593.)

[17] Xu, M. M.; Guo, C.; Hu, G. F.; Xu, S. Y.; Wang, L. Y. Chin. J. Chem. 2018, 36, 25.

[18] Yang, L. M.; Liu, B.; Li, N., Tang, B. Acta Chim. Sinica 2017, 75, 1047 (in Chinese). (杨立敏, 刘波, 李娜, 唐波, 化学学报, 2017, 75, 1047.)

[19] Wang, S. J.; Li, C. W.; Li, J.; Chen, B.; Guo, Y. Acta Chim. Sinica 2017, 75, 383 (in Chinese) (王少静, 李长伟, 李锦, 陈邦, 郭媛, 化学学报, 2017, 75, 383.)

[20] Theaker, B. J.; Hudson, K. E.; Rowell, F. J. Forensic Sci. Int. 2008 $174,26$.

[21] Domínguez, G.; Westphal, A. J.; Phillips, M. L. F.; Jones, S. M. Astrophys. J. 2003, 592, 631.

[22] Dantas, T. N. C.; Neto, A. A. D.; Moura, M. C. P. A.; Neto, E. L. B.; Forte, K. R; Leite, R. H. L. Water Res. 2003, 37, 2709.

[23] Yang, Y. Y.; Han, F. J.; Zhang, W. D.; Dai, Y. Y. Environ. Chem. 1998, 17, 19 (in Chinese). (杨义燕，韩扶军，张旺德，戴猷元，环境化学, 1998, 17, 19.)

[24] Zhang, T.; Yuan, H.; Wang, S. Q.; Guo, X. D.; Hu, R.; Li, Y.; Yang, G. Q. RSC Adv. 2017, 7, 32861 .

[25] Wang, Z.; Dai, Z.; Wu, J; Zhao, N.; Xu, J. Adv. Mater. 2013, 25, 4494.

[26] Huber, L.; Zhao, S. Y.; Malfait W. J.; Vares, S.; Koebel, M. M. Angew. Chem. Int. Ed. 2017, 56, 4753.

[27] Gao, M.; Tang, B. Z. ACS Sens. 2017, 2, 1382.

[28] Chen, M.; Sun, J. Z.; Qin A. J.; Tang, B. Z. Chin. Sci. Bull. 2016, 61, 304 (in Chinese). (陈明, 孙景志, 秦安军, 唐本忠, 科学通报, 2016, 61, 304.)

[29] Mei, J.; Leung, N. L. C.; Kwok, R. T. K.; Lam, J. W. Y.; Tang, B. Z. Chem. Rev. 2015, 115, 11718.

[30] Liu, C. X.; Zou, G. R.; Peng, S.; Wang, Y. F.; Yang, W.; Wu, F.; Jiang, Z. R.; Zhang, X.; Zhou, X. Angew. Chem., Int. Ed. 2018, 57, 9689.

[31] Plata, D. L.; Briones, Y. J.; Wolfe, R. L.; Carroll, M. K.; Bakrania, S. D.; Mandel, S. G.; Anderson, A. M. J. Non-Cryst. Solids 2004, $350,326$. 